\title{
Oxygen Activation in Lipoxygenase Model Reaction Studied with Density Functional Theory
}

\author{
Yuzo Nishida ${ }^{1}$ \\ ${ }^{1}$ Medical Research Institute, Kanazawa Medical University, Uchinada-machi 1-1, Japan \\ Correspondence: Yuzo Nishida, Medical Research Institute, Kanazawa Medical University, Uchinada-machi 1-1, \\ Kahoku-gun, Ishikawa Prefecture, 920-0293, Japan. E-mail: nsd-2210@kanazawa-med.ac.jp
}

Received: April 10, 2012 Accepted: May 3, 2012 Online Published: July 13, 2012

doi:10.5539/ijc.v4n4p1 URL: http://dx.doi.org/10.5539/ijc.v4n4p1

\begin{abstract}
Density Functional Computational studies were performed for the systems containing an iron (III) complex with (cyclam), 1,4-pentadiene and oxygen, where cyclam represents 1,4,8,11-tetraazacyclotetradecane. The calculations have indicated that bond formation between oxygen and carbon atom of the 1,4-pentadiene anion where methylene-proton is removed, occurs in the presence of the $\mathrm{Fe}^{\mathrm{III}}$-(cyclam) complex, giving the hydroperoxy derivative of the 1,4-pentadiene. Based on these results it seems quite reasonable to assume that deprotonation of the methylene proton should be a trigger for the peroxidation of the 1,4-pentadiene moiety and the electron transfer reaction between iron(III) atom and the substrate is unnecessary in the lipoxygenases.
\end{abstract}

Keywords: lipoxygenase model reaction, oxygen activation, DFT calculation

\section{Introduction}

Lipoxygenases are a class of nonheme iron oxygenases that catalyze the conversion of arachidonic acid and other polyunsaturated fatty acids to their hydroperoxy derivatives (see Scheme I) (Brash,1999; Goffa \& Brash, 2004; Oldham, Brash, \& Newcomer, 2005). The products are involved in a series of biological events such as inflammation, cell development, and differentiation.

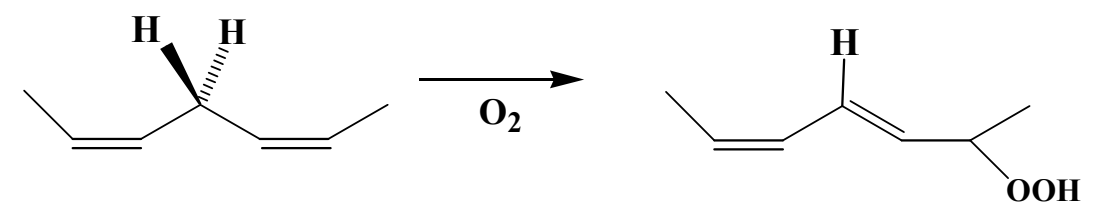

Scheme I

Lipoxygenases are expressed in some plant and animal tissues in high levels. The proteins have a single polypeptide chain with a molecular mass of $\sim 75-80 \mathrm{kDa}$ in animals and $\sim 94-104 \mathrm{kDa}$ in plants and have a $\mathrm{N}$-terminal $\beta$-barrel domain and a large catalytic domain containing a single atom of non-heme iron. The metal is liganded to conserved histidines and to the carboxyl group of a conserved isoleucine at the C-terminal of the protein. As the iron is non-heme of +2 oxidation state when isolated (inactive form), lipoxygenases appear virtually colorless to the eye, but oxidation to the ferric enzyme is required for catalysis (Brash, 1999).

There are several available crystal structures, of which three are of the arachidonate 15-lipoxygneases, soybean L-1, and rabbit reticulocyte 15-LOX, and the fourth, soybean L-3, is a catalyst of nonspecific peroxidation (Brash, 1999; Goffa \& Brash, 2004; Oldham et al., 2005). Many authors have believed that after the initial hydrogen abstraction by a ferric ion (formation of a radical species, L - and ferrous ion in Scheme II), antarafacial oxygenation at one end or the other of the activated pair of double bonds (pentadienes) gives product (Brash, 1999). Very recently, importance of proton-coupled electron transfer in soybean lipoxygenase was proposed, and many papers to support the above mechanism have appeared (Schenk, Neidig, Zhou, Holman, \& Solomon, 2003; Hatcher, Soudackov, \& H-Schiffer, 2007). But, there is no consensus on how substrate gains access to the metal center or any definite information on substrate bonding, especially how the oxidation of ferrous to ferric occurs, and on the reason why the oxidation of ferrous to ferric is necessary. 


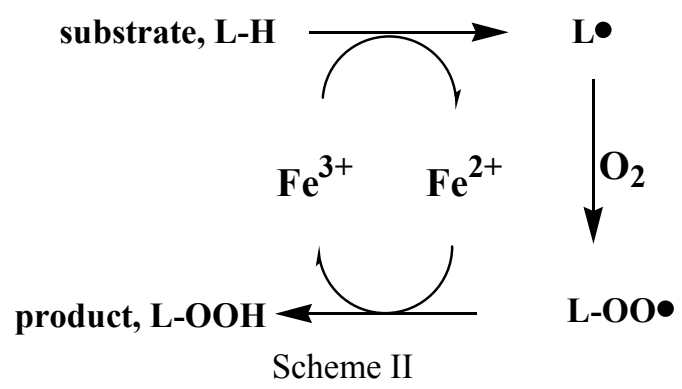

In our previous papers, we have reported that the monomeric trans-[Fe $\left.{ }^{\mathrm{III}}(\mathrm{cyclam}) \mathrm{Cl}_{2}\right]^{+}$complex can catalyze the peroxidation of linolenic acid under an aerobic condition without the change of oxidation state of iron(III) ion, where (cyclam) demonstrates 1,4,8,11-tetraazacyclotetradecane (Nishida \& Tanaka, 1994). Based on the fact that linolenic acid and oxygen interact under an aerobic condition to form a charge-transfer complex (Mulliken \& Pearson, 1969), which was verified by the absorption spectral study (Halliwell \& Gutteridge, 1985), we proposed a new mechanism for oxygen activation in the presence of iron(III) ion, i.e., at first the interaction between an iron(III) ion and the charge-transfer complex consisted of linolenic acid and triplet oxygen occurs through the interaction between the unpaired electrons of triplet oxygen and an iron(III) ion of high spin-type. This should lead to formation of ternary complex among iron(III) compound, triplet oxygen and linolenic acid as illustrated in Scheme III, giving the product (Nishida \& Tanaka, 1994). It should be noted here that triplet oxygen interacting with unpaired electron of transition metal ions acts as a singlet oxygen $\left({ }^{1} \Delta_{\mathrm{g}}\right)$ towards several organic compounds (Nishida \& Tanaka, 1994; Nishida, Tanaka, \& Okazaki, 1994; Nishida et al., 1995; Nishida, 2004), and that the change of the oxidation state of $\mathrm{Fe}^{3+}$ to the ferrous state $(+2)$ is not necessary for the formation of hydroperoxy-derivarives, because both the high- and low-spin type iron(III) ion have unpaired d-electrons. In this study we have performed density functional computational study to validate our mechanism on oxygen activation in the $\mathrm{Fe}(\mathrm{III})$-(cyclam) system and several lipoxygenase models.

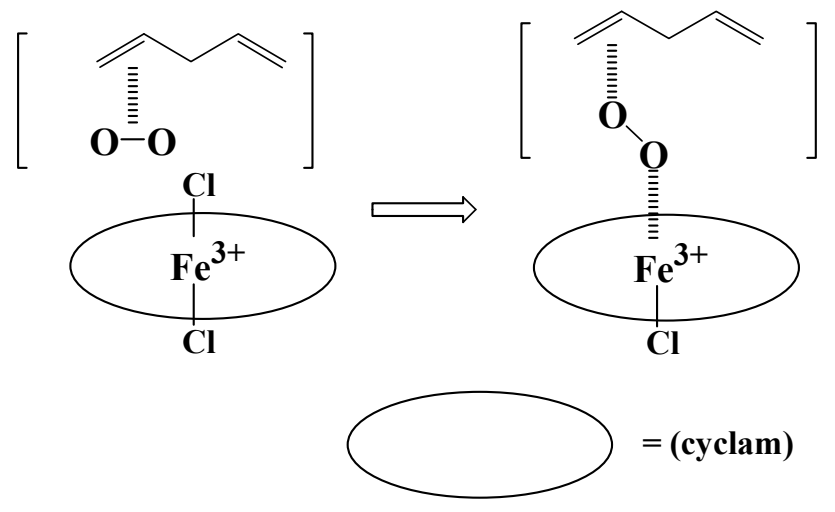

Scheme III

\section{Calculations}

All the calculations were performed with the Gaussian03suite (Frisch et al., 2004). For the present study, we used density functional theory(DFT), in particular, Becke's three-parameter hybrid exchange functional(B3) combined with Lee-Yang-Parr's (LYP) correlation functional, known as B3LYP (Khavrutskii, Musaev, \& Morokuma, 2003). Throughout this work, we used double- $\zeta$ LANL2DZ (D95V) basis set with associated Hay-Wadt nonrelativistic core potential. All geometries have been fully optimized without any symmetry constrains at the unrestricted B3LYP/LANL2DZ level.

A model for the lipoxygenase was constructed with a Fe(III) ion, three imidazole molecules, acetate anion, acetoamide, and water molecule, which is based on the structure of Soybean LOX-1 (see Figure 1) (Boyington, Gaffney, \& Amzel, 1993), and optimized by DFT calculation. In the DFT calculations, the charge transfer complex consisted of oxygen and 1,4-pentadine was brought to close to the iron(III) ion with the removal of water molecule from the iron(III) coordination sphere. The removal of the water molecule may be induced by the energy stabilization due to spin-pairing between unpaired electrons of iron atom and oxygen molecule, which should be promoted through the orbital interaction including the orbitals of substrate (Nishida, 2001; Nishida, 
2004).

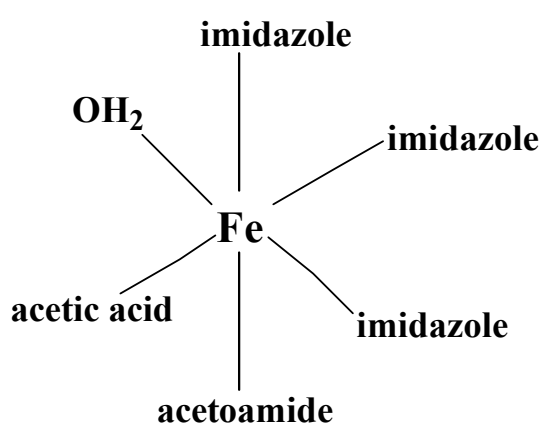

Figure 1. Coordination site around iron atom in the lipoxygenase model compound used in this study

\section{Results}

At first, we have confirmed that DFT calculations used in this study can realize the reactions specific for singlet oxygen $\left({ }^{1} \Delta_{\mathrm{g}}\right)$ as illustrated in Scheme IV.

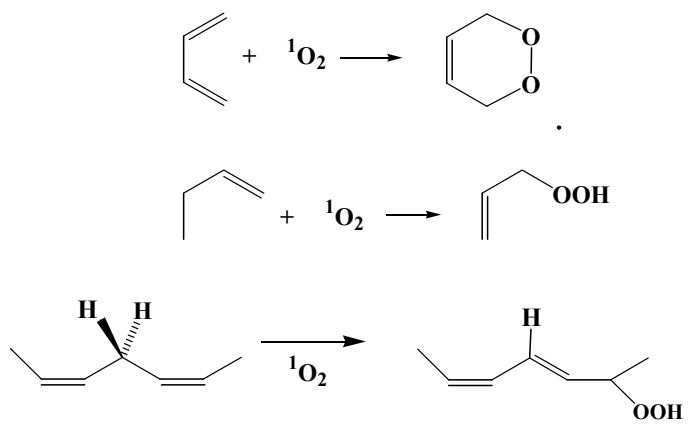

Scheme IV

Next, we have done the DFT calculations on the trans-[ $\mathrm{Fe}^{\mathrm{III}}$ (cyclam) $\mathrm{Cl}($ charge-transfer complex $\left.)\right]^{+}$system in Scheme-III, in order to clarify the conditions necessary for the bonding between oxygen and 1,4-pentadine (see right side of Scheme-III), where a charge-transfer complex is consisted of linolenic acid and triplet oxygen molecule. Our DFT calculations do not support the bond formation between oxygen and linolenic acid in the ternary complex, trans-[ $\mathrm{Fe}^{\mathrm{III}}(\mathrm{cyclam}) \mathrm{Cl}($ charge-transfer complex $\left.)\right]^{+}$system. However, it has become apparent that the binding between the oxygen molecule and the carbon atom of 1,4-pentadiene moiety occurs when one proton of the methylene part of the 1,4-pentadiene moietyis removed (see Scheme V). The resulted Fe(III) complex containing a hydroperoxyl-derivative (right side in the Scheme-V) may be a low-spin type(structureof the optimized finalspecies is illustrated in Figure 2), which was verified in the ESR spectral study on this system (Nishida \& Tanaka,1994). In our system in Scheme V, a proton-acceptor should be carboxylate-group of the linolenic acid present in the solution, and it seems quite reasonable to assume that deprotonation of the methylene proton may be a trigger for the preoxidation of the 1,4-pentadiene moiety.

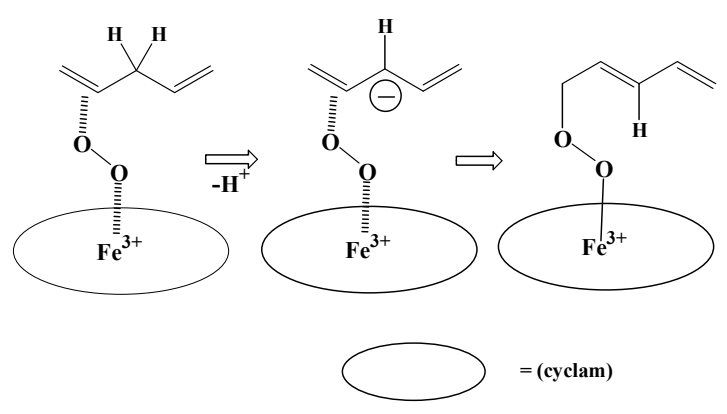

Scheme V 


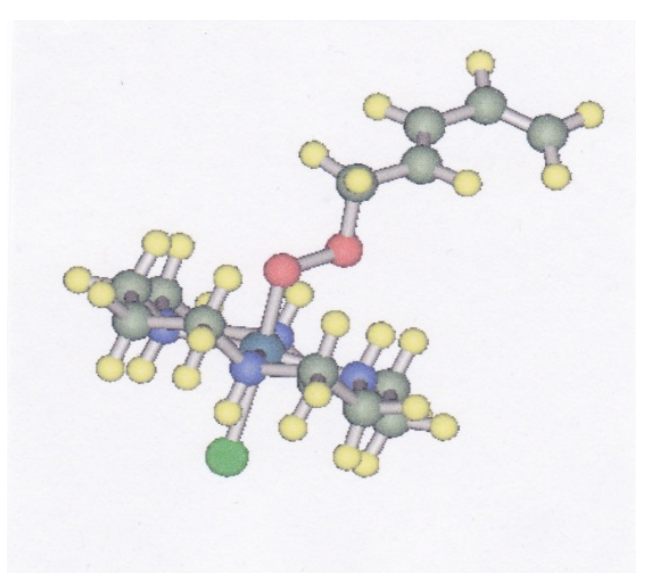

Figure 2. Optimized structure of Fe(cyclam)Cl(5-peroxy-1,3-pentaenediene): dark green, iron; green, chloride; red, oxygen; blue, nitrogen; pale green, carbon; yellow, hydrogen atom

DFT calculations also have revealed that the similar bond formation between the oxygen molecule and the carbon atom of 1,4-pentadiene moiety proceeds in the models of lipoxygenase(Figure 1) for both the $\mathrm{Fe}(\mathrm{II})$ (low-spin) and $\mathrm{Fe}$ (III) (high-spin) states when the 1,4-pentadiene moiety is deprotonated, and the optimized structure of the resulted ferric state-model containing hydroperoxy-derivative of 1,4-pentadiene is illustrated in Figure 3 ; this should correspond to a purple Fe ${ }^{\text {III }}$-OOL intermediate observed in SLO (Brash, 1999). As the hydroperoxy-derivative of unsaturated fatty acid has been used to obtain a ferric state lipoxygenase from a ferrous state in vitro (Goffa et al., 2005), it seems quite likely that the oxidation of the ferrous state to a ferric state proceeds through the reaction between the hydroperoxy-derivative formed at the first stage and Fe(II) ion in the native lipoxygenase, and the ferric state lipoxygenase thus formed should play an important role to produce the hydroperoxy-derivatives of fatty acid after the formation of iron(III) lipoxygenase.

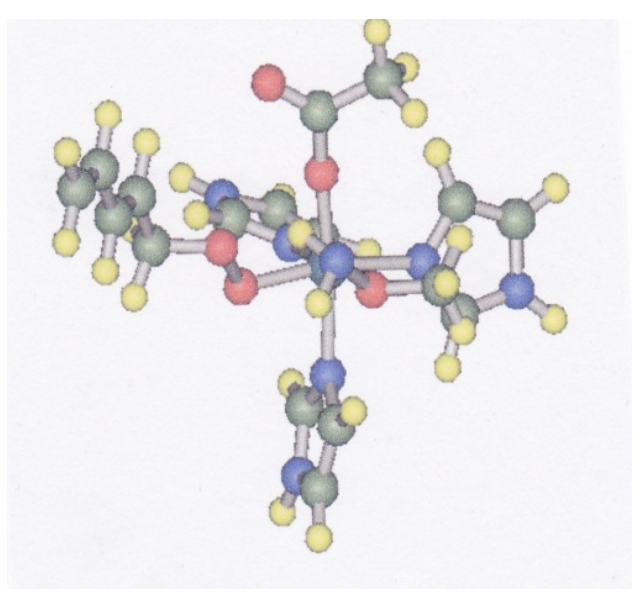

Figure 3. Optimized structure of (5-peroxy-1,3-pentaenediene)-Fe(III) compound of the lipoxygenase model compound (high-spin): dark green, iron; red, oxygen; blue, nitrogen; pale green, carbon; yellow, hydrogen atom

\section{Conclusion}

DFT calculations have indicated that bond formation between oxygen and carbon atom of the 1,4-pentadiene anion where methylene-proton is removed, occurs in the presence of the $\mathrm{Fe}^{\mathrm{III}}$-(cyclam) complex, giving the hydroperoxy derivative of the 1,4-pentadiene. Based on these results it seems quite reasonable to assume that deprotonation of the methylene proton should be a trigger for the peroxidation of the 1,4-pentadiene moiety and the electron transfer reaction between iron(III) atom and the substrate is unnecessary in the lipoxygenases.

Present results suggest that in the reactions of native lipoxygenase the presence of a proton acceptor, possibly the oxygen atoms of the protein residuals, should be necessary, and the position of the proton-acceptor may control the chirality of the products. The interaction between Fe(III) ion and oxygen molecule in the charge-transfer 
complex may be stronger than that in the corresponding Fe(II) case, and the deprotonation of the methylene hydrogen atom (in Scheme V) may be more facile in the system containing Fe(III) ion and charge-transfer complex of oxygen and unsaturated fatty acid. These may give reasonable reason why the oxidation of $\mathrm{Fe}(\mathrm{II})$ to a ferric state is necessary in the native lipoxygenase reaction.

The results obtained in this study strongly support the hypothesis that the electronic structure of the substrate determines the reactivity of the oxygen molecule in the oxygenasereactions, as pointed out in our previous works (Nishida, 2001, 2004; Nishida, Tanaka, \& Okazaki, 1994; Nishida, Ito, Okuno, \& Ohba, 1997).

Supplementary data: The positional data of the atoms in the optimized structures and the calculated results obtained in this study were deposited as Supplementary data (PDF file). These will be sent to all the chemists from the author on request.

\section{Acknowledgement}

The present author would like to thank Prof. H. Sakiyama at Yamagata University for his technical assistancein the DFT calculations and helpful discussions.

\section{References}

Boyington, J. C., Gaffney, B. J., \& Amzel, L. M. (1993). The three-dimensional structure of an arachidonic acid 15-lipoxygenase. Science, 260, 1482-1486. http://dx.doi.org/10.1126/science.8502991

Brash, A. R. (1999). Lipoxygenases: Occurrence, Functions, Catalysis, and Acquisition of Substrate. J. Biol. Chem., 274, 23679-23682.

Frisch, M. J., Trucks, G. W., Schlegel, H. B., Scuseria, G. E., Robb, M. A., Cheeseman, J. R., .. Pople, J. A. (2004). Gaussian, Inc., Wallingford CT.

Goffa, G., \& Brash, A. R. (2004). A single active site residue directs oxygenation stereospecifically in lipoxygenases: Stereocontrol is linked to the position of oxygenation. Proc. Natl. Acad. Sci. USA, 101, 15579-15584. http://dx.doi.org/10.1073/pnas.0406727101

Goffa, G., Imber, A. N., Maguire, B. C., Laxmikanthan, G., Schneider, C., Gaffney, B. J., \& Brash, A. R. (2005). On the relationships of substrate Orientation, Hydrogen abstraction, and product stereochemistry in single and double dioxygenation by soybean lipoxygenase-1 and its Ala542Gly mutant. J. Biol. Chem., 280, 38756-38766. http://dx.doi.org/10.1074/jbc.M504870200

Halliwell, B., \& Gutteridge, J. M. C. (1985). Free radicals in Biology and Medicine. Oxford University Press, chapter 4 .

Hatcher, E., Soudackov, A. V., \& H-Schiffer, S. (2007). Proton-coupled electron transfer in soybean lipoxygenase: Dynamical behavior and temperature dependence of kinetic isotope effects. J. Amer. Chem. Soc., 129, 187-196. http://dx.doi.org./10.1021/ja0667211

Khavrutskii, I. V., Musaev, D. G., \& Morokuma, K. (2003). Insights into the structure and reactivity of aceroperoxo complexes in the Kochi-Jacobson-Katsuki Catalytic system.A density functional study. J. Amer. Chem. Soc., 125, 13879-13889. http://dx.doi.org/10.1021/ja0343656

Mulliken, R. S., \& Pearson, W. B. (1969). Molecular Complex. New York: Wiley-Interscience.

Nishida, Y., \& Tanaka, N. (1994). Chemical Mechanism of oxygen activation by trans-[ $\mathrm{FeCl}_{2}$ (cyclam)] in the presence of reducing agents such as aliphatic aldehyde or linolenic acid. J. Chem. Soc. Dalton Trans., 2805-2809. http://dx.doi.org/10.1039/DT9940002805

Nishida, Y., Tanaka, N., \& Okazaki, M. (1994). Chemical mechanism of olefin oxygenation reaction catalyzed by bis(acetylacetonato)nickel(II) or cobalt(II) compounds in the presence of reducing agents. Polyhedron, 13, 2245-2250. http://dx.doi.org/10.1016/S0277-5387(00)88131-0

Nishida, Y., Tanaka, N., Yamazaki, A., Tokii, T., Hashimoto, K., Ide, K., \& Iwasawa, K. (1995). Novel reactivity of dioxygen molecule in the presence of a manganese(II) complex and reducing agents. Inorg. Chem., 34, 3616-3620. http://dx.doi.org/10.1021/ic00118a008

Nishida, Y., Ito, S., Okuno, T., \& Ohba, S. (1997). New insights into reaction of iron(III)-peroxide adduct with alkanes; an alternative model for cyctochrome P-450 and methane monooxygenase. Z. Naturforsch., 52C, 615-622.

Nishida, Y. (2001). Negligible ability of oxygen and peroxide in activation by Al(III) ion is essential for Al(III)-induced neurodegeneration. Z. Naturforsch., 56C, 865-871. 
Nishida, Y. (2004). Oxidative stress and neurodegeneration. Med Hypothesis Res, 1, 227-245. Retrieved from http://www.journal-mhr.com/PDF_Files/vol_1_4/1_4_PDFs/1_4_2.pdf

Oldham, M. L., Brash, A. R., \& Newcomer, M. E. (2005). Insights from the X-ray Crystal Structure of Coral 8R-Lipoxygenase. J. Biol. Chem., 280, 39545-39552. http://dx.doi.org/10.1074/jbcM506675200

Schenk, G., Neidig, M. L., Zhou, J., Holman, T. R., \& Solomon, E. I. (2003). Spectroscopic characterization of soybean lipoxygenase-1 mutants; the role of second coordination sphere residues in the regulation of enzyme activity. Biochemistry, 42, 7294-7302. http://dx.doi.org/10.1021/bi027380g 